\title{
Atomistic origin of huge response functions at the morphotropic phase boundary of $(1-x) \mathrm{Na}_{0.5} \mathrm{Bi}_{0.5} \mathrm{TiO}_{3}-x \mathrm{BaTiO}_{3}$
}

\author{
K. Datta, ${ }^{1,{ }^{*}}$ A. Richter,${ }^{2}$ M. Göbbels, ${ }^{2}$ R. B. Neder, ${ }^{1}$ and B. Mihailova ${ }^{3, \dagger}$ \\ ${ }^{1}$ Department Kristallographie und Strukturphysik, Friedrich-Alexander-Universität Erlangen-Nürnberg, \\ Staudtstr. 3, Erlangen 91058, Germany \\ ${ }^{2}$ Department Mineralogie, Friedrich-Alexander-Universität Erlangen-Nürnberg, Schlossgarten 5a, Erlangen 91054, Germany \\ ${ }^{3}$ Department Geowissenschaften, Universität Hamburg, Grindelallee 48, Hamburg 20146, Germany \\ (Received 13 March 2014; revised manuscript received 7 August 2014; published 28 August 2014)
}

\begin{abstract}
The ferroelectric solid solution $(1-x) \mathrm{Na}_{0.5} \mathrm{Bi}_{0.5} \mathrm{TiO}_{3}-\mathrm{BaTiO}_{3}(\mathrm{NBT}-x \mathrm{BT})$ is a promising material to substitute for the environmentally undesired $\mathrm{Pb}$-based ferroelectrics. The strong enhancement of the dielectric permittivity and piezoelectric coefficients near the morphotropic phase boundary (MPB) in ferroelectric $A B \mathrm{O}_{3}$-type solid solutions is commonly related to the existence of a single or several long-range-ordered phases resulting in a complex nanodomain pattern. Here, NBT- $x$ BT is studied by Raman scattering and complementary synchrotron $\mathrm{X}$-ray total elastic scattering to follow the temperature evolution of the mesoscopic-scale structural transformations for $x$ below, at, and slightly above $x_{\mathrm{MPB}}$. The most remarkable result is that at $x \sim x_{\mathrm{MPB}}$ the phonon mode involving vibrations of both off-centered A-site Bi and B-site Ti experiences strong softening and damping near the triplepoint temperature in the phase diagram, in strong contrast to the compounds with $x<x_{\mathrm{MPB}}$ or $x>x_{\mathrm{MPB}}$. The chemically enhanced coupling between the Bi and Ti subsystems at $x_{\mathrm{MPB}}$ is facilitated by the subtle disturbance of the coupling processes within the Bi subsystem induced by Ba at $x_{\mathrm{MPB}}$. These mesoscopic-scale structural and dynamic phenomena seem to be significant for the enhanced response functions at the MPB in NBT- $x$ BT.
\end{abstract}

DOI: 10.1103/PhysRevB.90.064112 PACS number(s): 77.84.-s, 78.30.-j, 77.80.B-, 61.05.C-

\section{INTRODUCTION}

Perovskite-type $\left(\mathrm{ABO}_{3}\right)$ materials with transition elements on the $\mathrm{B}$ site are the best choice to produce multifunctional ferroelectrics (FEs) with tunable properties, due to the flexibility of the perovskite framework when accommodating various chemical elements on the cationic A and/or B sites. Lead-based FE solid solutions exhibit outstanding dielectric, piezoelectric, pyroelectric, and optoelectric properties, but they have become undesired for future exploration because their production and consequent waste storage are environmentally unfriendly. As a consequence, a huge amount of research is currently being dedicated to find alternatives to $\mathrm{Pb}$-based systems with comparable properties. In this context, Bi-based systems have been anticipated as a suitable replacement, as $\mathrm{A}$-site $\mathrm{Bi}^{3+}$ has a stereochemically active $6 s^{2}$ lone pair similar to A-site $\mathrm{Pb}^{2+}$. Thus, Bi-based FE solid solutions have the great potential to be used in high-performance ecologically friendly devices, which has inspired extensive structural studies of these materials in order to better understand the structure-property relations. In particular, the solid solution $(1-x) \mathrm{Na}_{0.5} \mathrm{Bi}_{0.5} \mathrm{TiO}_{3}-x \mathrm{BaTiO}_{3}$ (NBT- $x$ BT) has received much attention due to its similarities with $\mathrm{PbZr}_{1-x} \mathrm{Ti}_{x} \mathrm{O}_{3}$ and $(1-x) \mathrm{PbB}_{1 / 3}^{\prime} \mathrm{B}_{2 / 3}^{\prime \prime} \mathrm{O}_{3}-x \mathrm{PbTiO}_{3}$ in terms of the existence of morphotropic phase boundary (MPB) with weak temperature dependence and strong enhancement of the dielectric permittivity and piezoelelctric response for $x \sim x_{\mathrm{MPB}}$ [1,2]. Additionally, NBT- $x$ BT exhibits a very rich phase diagram constructed on the basis of dielectricpermittivity anomalies and polarization hysteresis loop [1-3], which for low $x$ and high $T$ mismatches the observed changes in the average structure [4]. The nanodomain

\footnotetext{
*kaustuv.datta@physik.uni-erlangen.de

†boriana.mihailova@uni-hamburg.de
}

structure near $x_{\mathrm{MPB}}$ deduced from electron diffraction has been controversially reported to be monoclinic [5] or tetragonal double-perovskite [6-8]. Furthermore, similar to $(1-x) \mathrm{PbB}_{1 / 3}^{\prime} \mathrm{B}_{2 / 3}^{\prime \prime} \mathrm{O}_{3}-x \mathrm{PbTiO}_{3}$, NBT- $x \mathrm{BT}$ exhibits a relaxor behavior for $x \leqslant x_{\mathrm{MPB}}$ [9-11], i.e. existence of nonstatic polar nanoregions with a size insufficient to be considered as long-range ordered FE domains. All this highlights the importance of ferroic coupling on the mesoscopic scale and calls for the application of nonconventional methods sensitive to the local structure and dynamics.

The considerable deviation of the short- and intermediaterange order in pure NBT from the long-range order of the average structure was demonstrated by recent $\mathrm{x}$-ray absorption spectroscopy (XAS) [12] and neutron total elastic scattering (TES) $[13,14]$. Both methods revealed that (i) the off-centering of $\mathrm{B}$-site $\mathrm{Ti}^{4+}$ cations is only slightly influenced by temperatures and persists in the high-temperature paraelectric phase at least up to $870 \mathrm{~K}$, and (ii) the off-centering of A-site $\mathrm{Bi}^{3+}$ is considerably larger and less dispersed than that of A-site $\mathrm{Na}^{+}$cations. Neutron pair distribution function (PDF) analysis demonstrated that the $\mathrm{B}$-site $\mathrm{Ti}^{4+}$ cations are rhombohedrally displaced at all studied temperatures between 766 and $10 \mathrm{~K}$, while the $\mathrm{A}$-site $\mathrm{Bi}^{3+}$ cations are tetrahedrally displaced at $766 \mathrm{~K}$, form two distinct groups of monoclinically displaced cations between 473 and $298 \mathrm{~K}$, and all are predominantly rhombohedrally off-centered at 10 $\mathrm{K}$ [14]. The complex temperature evolution of the off-centered $\mathrm{Bi}^{3+}$ displacements indicates that the $\mathrm{Bi}^{3+}$ coupling is the key factor for the multiple phase-transition sequence in pure NBT, and its disturbance by $\mathrm{Ba}^{2+}$ may be the main reason for the enhanced dielectric and piezoelectric properties at $x \sim x_{\mathrm{MPB}}$ in NBT- $x$ BT.

Raman spectroscopy is a method sensitive to local structural transformations and, thus, studies of the temperature dependence of phonon modes involving $\mathrm{Bi}$ vibrations can 
provide valuable information about the formation of the FE state near MPB in NBT- $x$ BT. $\mathrm{Bi}^{3+}$ is similar to $\mathrm{Pb}^{2+}$ in mass and outermost electron shell and, therefore, Raman peaks generated by Bi-localized modes phonon modes (involving mainly $\mathrm{Bi}$ vibrations) in $\mathrm{ABO}_{3}$-type materials may appear near $50 \mathrm{~cm}^{-1}$, like the phonon modes involving mainly $\mathrm{Pb}$ vibrations in $\mathrm{Pb}$-based perovskite-type solid solutions [15-17] as well as like Bi-localized modes in complex bismuth oxides of other structure types [18,19]. Raman scattering near $50 \mathrm{~cm}^{-1}$ was indeed detected in pure NBT [20], but surprisingly, the majority of reported Raman spectroscopic studies of Bi-based perovskite-type ferroelectrics did not consider the important spectral range below $100 \mathrm{~cm}^{-1}$ [21-26]. Analyses on the behavior of the Raman scattering near $50 \mathrm{~cm}^{-1}$ in Bi-based ferroelectric solid solutions are scarce [27] and, to the best of our knowledge, no such studies were conducted on NBT-based solid solutions with variable content of Bi.

In this paper the temperature evolution of Raman-active phonon modes between 15 and $1200 \mathrm{~cm}^{-1}$ in three NBT$x$ BT compounds with $x=0<x_{\mathrm{MPB}}, x=0.06 \sim x_{\mathrm{MPB}}$, and $x=0.08>x_{\mathrm{MPB}}$ are comparatively analyzed to elucidate the differences between the mesoscopic-scale transformation processes at and aside from MPB. The Raman scattering data are complemented with temperature-dependent $\mathrm{x}$-ray TES.

\section{EXPERIMENTAL DETAILS}

\section{A. Sample preparation}

Ceramic samples of NBT- $x$ BT were produced following the conventional solid-state synthesis route. Starting materials $\left(\mathrm{Na}_{2} \mathrm{CO}_{3}, \mathrm{Bi}_{2} \mathrm{O}_{3}, \mathrm{TiO}_{2}\right.$, and $\left.\mathrm{BaCO}_{3}\right)$ were first thoroughly mixed by wet ball-milling in ethanol media for about $24 \mathrm{~h}$ before the dried powders were heat processed. Three stages of heating with intermediate grinding were performed for each sample. Undoped NBT was first calcined at $1073 \mathrm{~K}$ for $6 \mathrm{~h}$, then at $1273 \mathrm{~K}$ for $4 \mathrm{~h}$, and finally sintered at $1373 \mathrm{~K}$ for $2.5 \mathrm{~h}$. The doped samples were calcined at $1173 \mathrm{~K}$ for $6 \mathrm{~h}$ and at $1323 \mathrm{~K}$ for $4 \mathrm{~h}$. They were finally sintered at $1423 \mathrm{~K}$ for $2.5 \mathrm{~h}$. The chemical compositions of the sintered samples were verified by wavelength-dispersive spectroscopy (WDS) analysis, using an electron probe microanalyzer JEOL JXA820 , with an acceleration voltage of $15 \mathrm{kV}$ and a probe current of $15 \mathrm{nA}$. Albite $\left(\mathrm{NaAlSi}_{3} \mathrm{O}_{8}\right)$, rutile $\left(\mathrm{TiO}_{2}\right)$, metallic $\mathrm{Bi}$, and $\mathrm{BaO}$ were used as standards for $\mathrm{Na}, \mathrm{Ti}, \mathrm{Bi}$, and $\mathrm{Ba}$, respectively.

\section{B. Raman scattering}

Raman spectra were collected with a Horiba Jobin-Yvon T64000 triple-grating spectrometer equipped with an Olympus BH41 microscope and a $50 \times$ long-working distance objective. The measurements were conducted on plate-shaped pellets of pressed powders in a backscattering geometry. The 514.5-nm line of an $\mathrm{Ar}^{+}$laser (Coherent 90C FreD) with a power density on the sample surface of $1.4 \mathrm{~kW} / \mathrm{mm}^{2}$ was used to excite the Raman scattering. The achieved spectral resolution was approximately $2 \mathrm{~cm}^{-1}$. At room temperature no polarization, orientation, or spatial dependence of the Raman spectra was detected, indicating that the average linear crystallite size is much smaller than the diameter of the laser spot on the sample surface, which was $\sim 2 \mu \mathrm{m}$. In situ temperature-dependent spectra were collected on cooling from 870 to $80 \mathrm{~K}$ using a Linkam THMS-E600 stage with a cooling rate of $10 \mathrm{~K} / \mathrm{min}$ and a temperature accuracy of $\pm 0.1 \mathrm{~K}$. The reversibility of the observed temperature-induced structural changes was verified by measuring the room-temperature spectrum at the end of each run. The measured spectra were corrected by the Bose-Einstein phonon occupation factor to eliminate the effect of temperature on the peak intensities. The temperaturereduced spectra were then fitted with pseudo-Voigt peak-shape functions $\{P V=q L+(1-q) G, q \in[0,1], L$ and $G$ stand for Lorentzian and Gaussian, respectively\} to determine the phonon wave numbers $\omega$, full widths at half maximum (FWHMs), integrated intensities $I$ normalized to the total spectrum profile areas, and the Lorentzian weight coefficients $q$. The choice of the peak shape function was justified by the fact that both local structural and chemical disorder are expected in solid solutions, which leads to phonon modes statistically dispersed in energy and results in a nonzero Gaussian contribution to the Raman peak shape.

\section{X-ray total elastic scattering}

Temperature-dependent powder diffraction data were collected on heating between 120 to $800 \mathrm{~K}$ at the BW5 beamline of the DORIS-III facility in Deutsches ElektronenSynchrotron (DESY) using an incident beam energy of $100 \mathrm{keV}(\lambda=0.12398 \AA)$, which provided a maximum reciprocal-space $Q$ value of $25 \AA^{-1}$. A flat panel detector (Perkin Elmer 1621) was used to record the two-dimensional (2D) powder rings. Powder samples were put in a quartz capillary of diameter $\sim 1.0 \mathrm{~mm}$ and then placed into a Linkam THMS600 cryofurnace. In situ 2D powder rings were collected on the fly with a temperature ramp of $2 \mathrm{~K} / \mathrm{min}$. The $2 \mathrm{D}$ images were integrated into typical one-dimensional (1D) powder patterns using the Fit2D software [28]. Final data corrections and Fourier transformations were performed using the PDFgetX3 software [29] to obtain the pair distribution functions.

\section{RESULTS AND DISCUSSION}

The temperature-reduced Raman spectra of NBT- $x$ BT with $x=0,0.06$, and 0.08 collected at 80 and $870 \mathrm{~K}$ are shown in Fig. 1. As can be seen, all three compounds exhibit pronounced Raman scattering at $870 \mathrm{~K}$, although they are all in a paraelectric state with symmetry of the average structure $P m \overline{3} m$ [4], in which no Raman-active modes are allowed (see Table I) [30]. If the A-site $\mathrm{Na}^{+}$and $\mathrm{Bi}^{3+}$ cations form a NaCl-type chemical order, the crystallographic unit cell would double [20], and the symmetry of the paraelectric phase would be $F m \overline{3} m$, in which one triply degenerate Raman-active mode localized in the B-site cations is allowed [20,30]. However, recent density functional theory calculations on pure NBT revealed that the most thermodynamically stable A-site cation configuration involves alternating planes of $\mathrm{Bi}$ and $\mathrm{Na}$ along one of the cubic $\langle 100\rangle$ directions rather than $1: 1$ alternations of $\mathrm{Bi}$ and $\mathrm{Na}$ atoms along all $\langle 100\rangle$ cubic directions [31], i.e. doubling of the perovskite structure due to $\mathrm{NaCl}$-type chemical order is hardly plausible even on the mesoscopic scale. Therefore, we attribute the Raman 


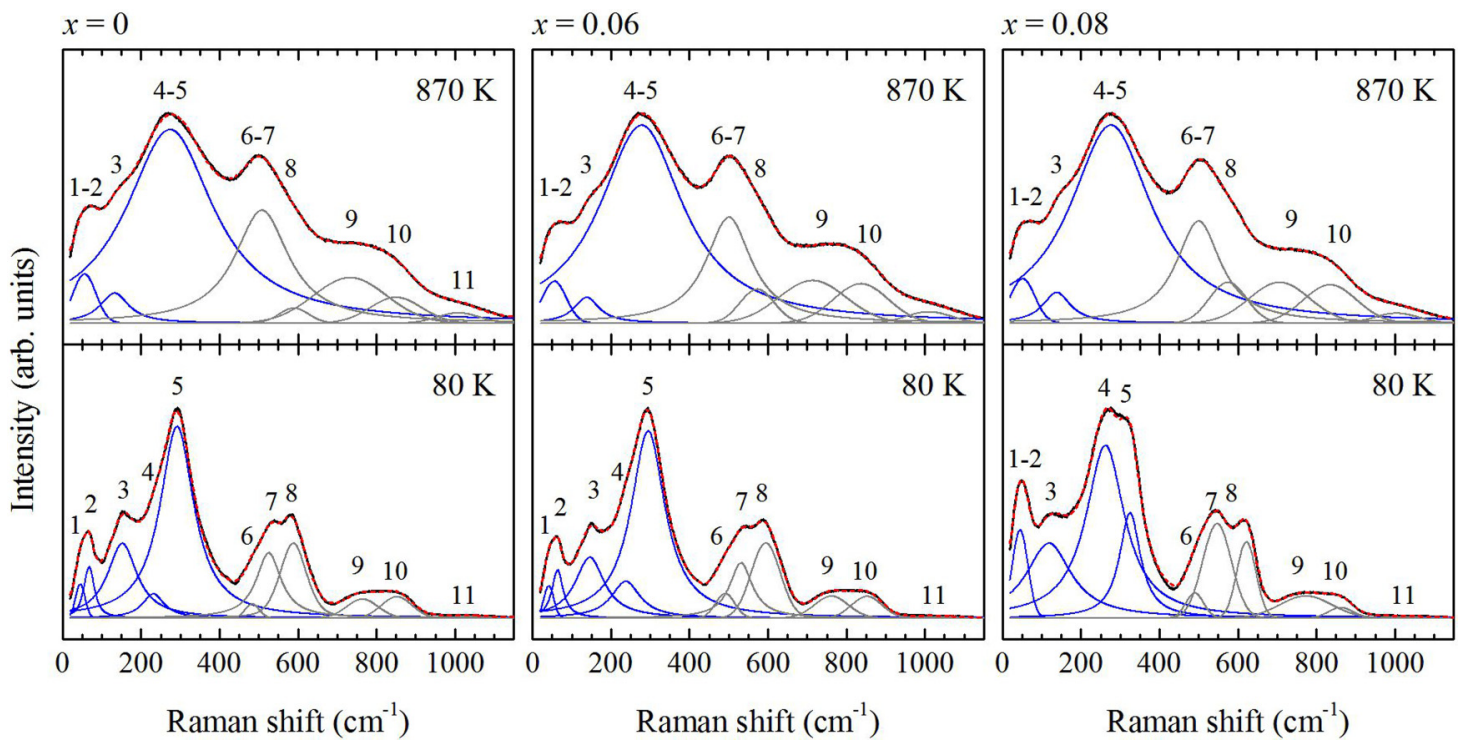

FIG. 1. (Color online) Raman spectra of NBT- $x$ BT with $x=0,0.06$, and 0.08 measured at 80 and $870 \mathrm{~K}$ (solid black lines), the fitting pseudo-Voigt peak functions (thin lines), and the resultant spectrum profiles (dashed red lines). The blue thin lines mark the Raman peaks for which the temperature evolution is further discussed. Note that the Gaussian contribution to the shape of peaks 3, 4, and 5 was negligibly small within the entire temperature range $80-870 \mathrm{~K}$.

scattering observed in the paraelectric state to local ferroic distortions, previously detected also by XAS [12] and neutron TES $[13,14]$.

The Raman spectra measured at $870 \mathrm{~K}$ are essentially the same for $x=0,0.06$, and 0.08 , but at $80 \mathrm{~K}$, the spectrum of NBT-0.08BT differs from the spectra of NBT and NBT-0.06BT in the shape of the scattering near 50,290 , and $570 \mathrm{~cm}^{-1}$ (see Fig. 1). To quantitatively evaluate the Raman data, we have fitted the spectrum profiles with pseudo-Voigt functions. For each compound, we first fitted the spectrum at $80 \mathrm{~K}$, and the number of included peaks was chosen following the criterion that the obtained uncertainty in the intensity $d I$ for each peak should be smaller than the corresponding intensity $I$. Then the same number of peaks was used as a starting model for the spectrum measured at the next higher temperature and, if for a certain peak, $d I$ was larger than $I$, that peak was removed. We also tried to fit the spectra using only Lorentzians, but that yielded worse $R^{2}$ and $\chi^{2}$ factors than fitting with pseudo-Voigt functions, indicating that for the majority of phonon modes, the Gaussian contribution to the peak shape due to the existence of structural and/or chemical disorder cannot be neglected. The Gaussian contribution was close to zero through the entire temperature range only for the peaks between $130-300 \mathrm{~cm}^{-1}$ (peaks 3-5 in Fig. 1). Such a strategy was entirely based on mathematical criteria as group-theory analysis was not very helpful because the resolved Raman peaks in the lowtemperature spectra apparently mismatched the number of fundamental Raman-active modes in the possible ferroelectric phases: $R 3 c, C c, P 4 b m$, for $x=0$ [4,32,33], $R 3 c, C c, P 4 b m$, $P 4 m m$ for $x=0.06$ [4-8], and P4mm for $x=0.08$ [4,34]. Group-theory analysis of the phonon modes at the Brillouinzone center of the corresponding phases is given in Table I. As can be seen in Table I, if possible two-mode behavior due to compositional disorder on the A site is neglected, the number of expected Raman peaks in $R 3 c, P 4 b m, C c$, and $P 4 m m$ are respectively $13,16,27$, and 8 , without taking into account the longitudinal optic (LO)-transverse optic (TO) effects, or $26,24,54$, and 14, respectively, considering both the LO and TO components related to the polar modes. It should be underlined that the vibrational states in polar crystals that are arbitrarily oriented to the direction of the incident photon are quasi-LO and quasi-TO phonons with mixed symmetry and wave numbers depending on the angle between the phonon propagation and the crystallographic polar axis [35]. This further complicates the application of symmetry analysis to Raman spectra of polycrystalline multidomain samples. Stable fits with satisfactory $d I / I$ could not be achieved using either of the predicted number of peaks for the range $15-950 \mathrm{~cm}^{-1}$ (the weak scatting near $1025 \mathrm{~cm}^{-1}$ labeled in Fig. 1 as peak 11 arises most probably from multiphonon scattering). Assuming two-mode behavior for the optical modes associated with A-site cations would lead to even larger number of expected peaks for $R 3 c, P 4 b m$, and $C c$ and would not influence the number of expected Raman peaks in $P 4 m m$ [see Table I and footnote (c)]. In fact, with the exception of the Raman scattering near $50 \mathrm{~cm}^{-1}$, the low-temperature spectra of NBT, NBT-0.06BT, and NBT-0.08BT exhibit the same number of resolved peaks (see Fig. 1). The Raman peaks in the range 400 $950 \mathrm{~cm}^{-1}$ most probably result from internal $\mathrm{BO}_{6}$ vibrations, i.e. the corresponding phonon modes are comprised mainly of oxygen vibrations. This Raman scattering persists at elevated temperatures, indicating that the $\mathrm{BO}_{6}$ are still ferroically deformed. The Raman scattering near $290 \mathrm{~cm}^{-1}$ (peaks 4 and 5 in Fig. 1) is also associated with distorted $B_{6}$ octahedra [36], but due to the lower energy range, the generating phonon modes should considerably involve vibrations of off-centered B-site cations. The latter assumption is strongly supported by the fact that similar Raman scattering appears at lower 
TABLE I. Crystal normal phonon modes in NBT- $x$ BT in plausible space-symmetry groups, determined using the online available utilities on the Bilbao Crystallographic server (http://www.cryst.ehu.es/) [30].

\begin{tabular}{|c|c|c|c|}
\hline Space group & Atom & Wyckoff position & Normal phonon modes at the Brillouin-zone center $\Gamma^{\mathrm{a}, \mathrm{b}, \mathrm{c}}$ \\
\hline$P m \overline{3} m$ & $\begin{array}{l}\mathrm{Na} / \mathrm{Bi} / \mathrm{Ba} \\
\mathrm{Ti} \\
\mathrm{O}\end{array}$ & $\begin{array}{l}1 b \\
1 a \\
3 d\end{array}$ & $\begin{array}{l}T_{1 u}(\text { ir }) \\
T_{1 u}(\text { ir }) \\
T_{2 u}(\text { ina })+2 T_{1 u}(\text { ir }) \\
\Gamma_{\text {total }}=T_{2 u}(\text { ina })+4 T_{1 u}(\text { ir }) \\
\Gamma_{\text {acoustic }}=T_{1 u} \\
\Gamma_{\text {optical }}=T_{2 u}(\text { ina })+3 T_{1 \mathrm{u}}(\text { ir })\end{array}$ \\
\hline$R 3 c$ & $\begin{array}{l}\mathrm{Na} / \mathrm{Bi} / \mathrm{Ba} \\
\mathrm{Ti} \\
\mathrm{O}\end{array}$ & $\begin{array}{l}2 a \\
2 a \\
6 b\end{array}$ & $\begin{array}{l}A_{1}(r, i r)+A_{2}(\text { ina })+2 E(r, i r) \\
A_{1}(r, i r)+A_{2}(\text { ina })+2 E(r, i r) \\
3 A_{1}(r, i r)+3 A_{2}(\text { ina })+6 E(r, i r) \\
\Gamma_{\text {total }}=5 A_{1}(r, i r)+5 A_{2}(\text { ina })+10 E(r, i r) \\
\Gamma_{\text {acoustic }}=A_{1}+E \\
\Gamma_{\text {optical }}=4 A_{1}(r, i r)+5 A_{2}(\text { ina })+9 E(\text { r,ir })\end{array}$ \\
\hline$P 4 b m$ & $\begin{array}{l}\mathrm{Na} / \mathrm{Bi} / \mathrm{Ba} \\
\mathrm{Ti} \\
\mathrm{O} 1 \\
\mathrm{O} 2\end{array}$ & $\begin{array}{l}2 b \\
2 a \\
2 a \\
4 c\end{array}$ & $\begin{array}{l}A_{1}(r, i r)+B_{2}(r)+2 E(r, i r) \\
A_{1}(r, i r)+A_{2}(\text { ina })+2 E(r, i r) \\
A_{1}(r, i r)+A_{2}(\text { ina })+2 E(r, i r) \\
2 A_{1}(r, i r)+A_{2}(\text { ina })+B_{1}(r)+2 B_{2}(r)+3 E(r, i r) \\
\Gamma_{\text {total }}=5 A_{1}(r, i r)+3 A_{2}(\text { ina })+B_{1}(r)+3 B_{2}(r)+9 E(r, i r) \\
\Gamma_{\text {acoustic }}=A_{1}+E \\
\Gamma_{\text {optical }}=4 A_{1}(r, i r)+3 A_{2}(\text { ina })+B_{1}(r)+3 B_{2}(r)+8 E(r, i r)\end{array}$ \\
\hline$C c$ & $\begin{array}{l}\mathrm{Na} / \mathrm{Bi} / \mathrm{Ba} \\
\mathrm{Ti} \\
\mathrm{O} 1 \\
\mathrm{O} 2 \\
\mathrm{O} 3\end{array}$ & $\begin{array}{l}4 a \\
4 a \\
4 a \\
4 a \\
4 a\end{array}$ & $\begin{array}{l}3 A^{\prime}(r, i r)+3 A^{\prime \prime}(r, i r) \\
3 A^{\prime}(r, i r)+3 A^{\prime \prime}(r, i r) \\
3 A^{\prime}(r, i r)+3 A^{\prime \prime}(r, i r) \\
3 A^{\prime}(r, i r)+3 A^{\prime \prime}(r, i r) \\
3 A^{\prime}(r, i r)+3 A^{\prime \prime}(r, i r) \\
\Gamma_{\text {total }}=15 A^{\prime}(r, i r)+15 A^{\prime \prime}(r, i r) \\
\Gamma_{\text {acoustic }}=2 A^{\prime}(r, i r)+A^{\prime \prime}(r, i r) \\
\Gamma_{\text {optical }}=13 A^{\prime}(r, i r)+14 A^{\prime \prime}(r, i r)\end{array}$ \\
\hline$P 4 m m$ & $\begin{array}{l}\mathrm{Na} / \mathrm{Bi} / \mathrm{Ba} \\
\mathrm{Ti} \\
\mathrm{O} 1 \\
\mathrm{O} 2\end{array}$ & $\begin{array}{l}1 a \\
1 b \\
1 b \\
2 c\end{array}$ & $\begin{array}{l}A_{1}(r, i r)+2 E(r, i r) \\
A_{1}(r, i r)+2 E(r, i r) \\
A_{1}(r, i r)+2 E(r, i r) \\
A_{1}(r, i r)+B_{1}(r)+2 E(r, i r) \\
\Gamma_{\text {total }}=4 A_{1}(r, i r)+B_{1}(r)+5 E(r, i r) \\
\Gamma_{\text {acoustic }}=A_{1}+E \\
\Gamma_{\text {optical }}=3 A_{1}(r, i r)+B_{1}(r)+4 E(r, i r)\end{array}$ \\
\hline
\end{tabular}

a $r$, Raman-active; ir, infrared-active; ina, inactive.

${ }^{\mathrm{b}}$ Optical phonon modes which are simultaneously Raman and infrared active have longitudinal (LO) and transverse (TO) component and hence, they may give rise to two Raman peaks.

${ }^{\mathrm{c}}$ Acoustic modes have a wave number approaching zero at the $\Gamma$ point and therefore they are commonly attributed to the atoms with the largest mass and/or weakest bounding to the surrounding atoms, i.e., to the A-site cations in the case of NBT- $x$ BT.

wave numbers in perovskite-type ferroelectrics with heavier cations on the $\mathrm{B}$ site, including $\mathrm{Nb}$ and $\mathrm{Ta}$ [15-17]. Thus, the persistence of the Raman peak near $290 \mathrm{~cm}^{-1}$ at high temperatures for all three NBT- $x$ BT compounds indicates the presence of off-centered Ti even in the paraelectric state, in full accordance with previous XAS [12] and TES studies [14]. The Raman scattering near $140 \mathrm{~cm}^{-1}$ in NBT-based solid solutions was previously ascribed to vibrations of A-site alkali cations, as a result of two-mode behavior of the mode localized in the A-site cations [36]. The arguments for such a peak assignment were the smaller mass of alkali elements as compared to the mass of $\mathrm{Bi}$, which may lead to a higher wave number component related to alkali-cation vibrations and a lower wave number component related to $\mathrm{Bi}$ vibrations, and the shift of this peak toward lower wave numbers when $\mathrm{K}$ substitutes for $\mathrm{Na}$ [36]. The latter, however, can be due to chemically induced structural alteration rather than a direct chemical effect on the A-cation vibrations. For example, the addition of Ba to NBT causes a rhombohedral-to-tetragonal change in the average structure, and the position of the peak near $140 \mathrm{~cm}^{-1}$ stepwise drops at $x_{\mathrm{MPB}}$ instead of gradually decreasing with the increase in the content of $\mathrm{Ba}$ [21], which unambiguously speaks in favor of an indirect chemically driven structural effect rather than a direct chemical effect. Furthermore, a similar Raman peak near $140 \mathrm{~cm}^{-1}$ is observed in perovskite-type ferroelectric solid solutions with only $\mathrm{Pb}$ or with both $\mathrm{Bi}$ and $\mathrm{Pb}$ on the A-site [15-17,26], which also emphasizes that this peak cannot be related to a phonon mode localized in light alkali elements on 


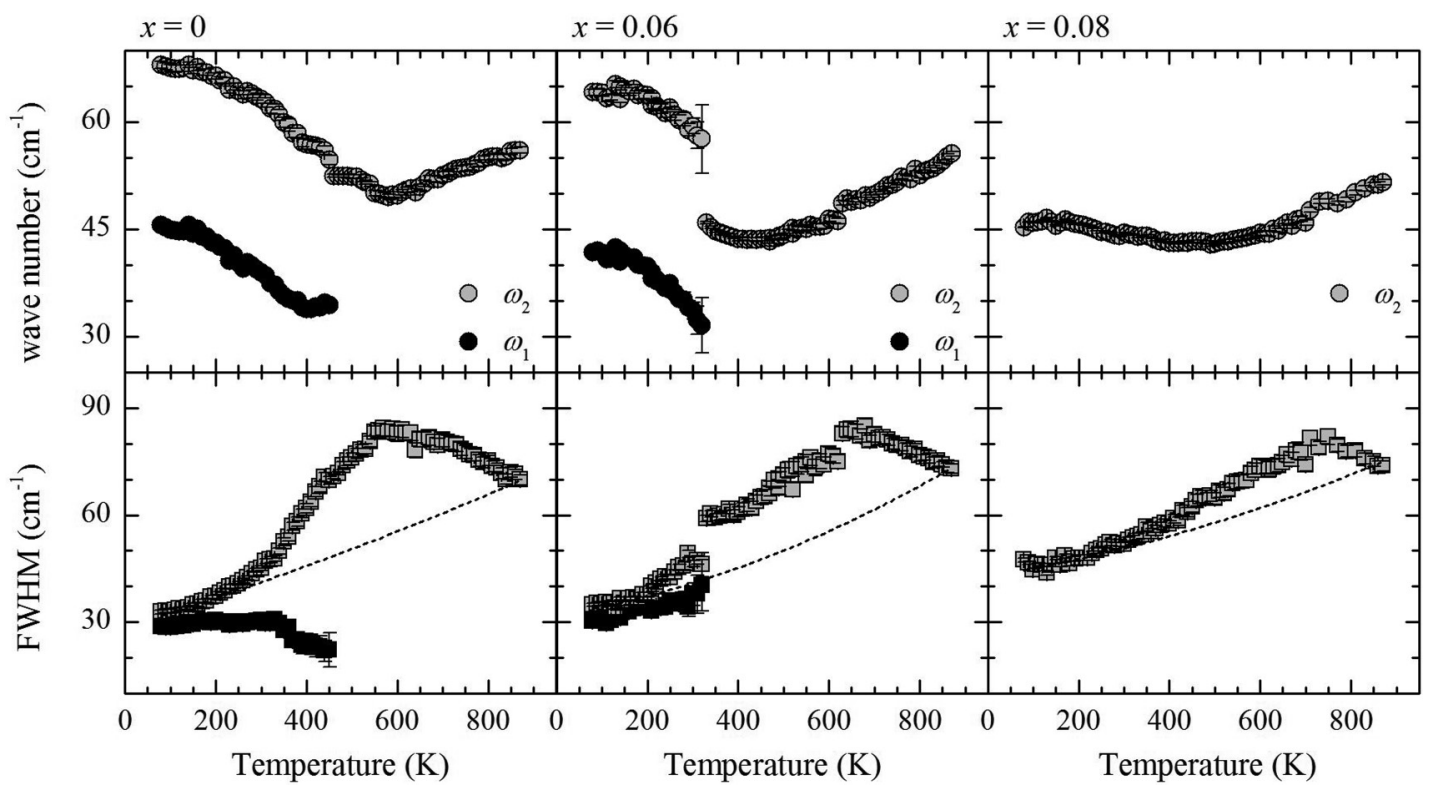

FIG. 2. Temperature evolution of the wave number $\omega$ and FWHM of Bi-localized mode in NBT- $x$ BT for $x=0,0.06$, and 0.08 ; gray and black symbols represent the higher energy and lower energy component, respectively. The dashed lines in the bottom plots are polynomial baselines of the datasets, representing the conventional decrease of phonon mode widths on cooling in the absence of phase transition.

the A site. By analogy with complex Pb-based perovskite-type ferroelectrics [15-17], we assign the Raman scattering at $\sim 140 \mathrm{~cm}^{-1}$ in NBT- $x$ BT to an $\mathrm{A}-\mathrm{BO}_{3}$ translation mode involving vibrations of both $\mathrm{A}$ - and B-site cations. Previous studies of $\mathrm{Pb}$-based relaxors and relaxor-ferroelectric solid solutions demonstrated that this Raman scattering mirrors the coupling processes between off-centered A- and B-site cations [15-17]. On the other hand, reverse Monte Carlo simulations of neutron TES of pure NBT [13] revealed that, due to the ionic type of $\mathrm{Na}-\mathrm{O}$ interactions, $\mathrm{Na}^{+}$tends to position at the center of the 12 surrounding $\mathrm{O}^{2-}$ anions, in strong contrast to $\mathrm{Bi}^{3+}$ which shows affinity to off-center. This, along with the relatively high degree of covalency of $\mathrm{Bi}-\mathrm{O}$ interactions and subsequently larger Raman cross section, implies that the Raman scattering near $140 \mathrm{~cm}^{-1}$ in NBT- $x$ BT is dominated by $\mathrm{Bi}^{-\mathrm{TiO}_{3}}$ vibrations and should be sensitive to coupling processes between off-centered $\mathrm{Bi}^{3+}$ and off-centered $\mathrm{Ti}^{4+}$ cations. The Raman scattering near $50 \mathrm{~cm}^{-1}$ of NBT- $x$ BT is attributed to phonon modes localized in A-site cations [27] and again due to the mixed ionic-covalent character of $\mathrm{Bi}-\mathrm{O}$ bonding versus the ionic type of $\mathrm{Na}-\mathrm{O}$ and $\mathrm{Ba}-\mathrm{O}$ interactions, it should be dominated by $\mathrm{Bi}$ vibrations. As expected, the wave number of this Raman scattering matches well that of Bi-localized modes in complex bismuth oxides [18,19] as well as of $\mathrm{Pb}$-localized modes in perovskite-type relaxors and relaxor-ferroelectric solid solutions [15-17]. For all three compounds studied here, the Raman peak near $50 \mathrm{~cm}^{-1}$ persists at elevated temperatures (see Fig. 1), although it is not symmetryallowed in $P m \overline{3} m$, indicating the presence of off-centered $\mathrm{Bi}^{3+}$ cations in the paraelectric state, in agreement with XAS [12] and TES [13,14].

Thus, we have focused our analysis on the temperature evolution of the Raman scattering near 50, 290, and $140 \mathrm{~cm}^{-1}$, which respectively reflects the coupling processes within the subsystem of off-centered $\mathrm{Bi}$, within the subsystem of offcentered $\mathrm{Ti}$, and between these two subsystems.

Figure 2 shows the temperature dependencies of the wave number $\omega$ and FWHM $\Gamma$ of the Bi-localized mode near $50 \mathrm{~cm}^{-1}$. For $x=0$ and 0.06 , this mode exhibits splitting at $T_{\text {split }}^{\omega_{1}-\omega_{2}} \sim 450$ and $\sim 370 \mathrm{~K}$, respectively. For $x=0.08$, splitting of the Bi-localized mode near $50 \mathrm{~cm}^{-1}$ could not be resolved, but the Lorentzian contribution $q$ becomes zero below $420 \mathrm{~K}$, in contrast to the case of $x=0$ and 0.06 for which the Lorentzian contribution of the higher energy components of the Bi-localized mode (peak 2 in Fig. 1) approaches unity in the temperature range where the mode exhibits splitting. This suggests that, if the content of $\mathrm{Ba}$ is above $x_{\mathrm{MPB}}$, the compositional disorder on the A site is too high to allow for two distinct low-temperature dynamical states of $\mathrm{Bi}$, at least down to $80 \mathrm{~K}$. For all three compounds studied, $\omega_{2}(T)$ exhibits a minimum accompanied by an excess of FWHM, which is most pronounced for $x=0$, indicating that the coupling processes within the subsystem of off-centered $\mathrm{Bi}$ are strongest in pure NBT. The temperature of the minimum of $\omega_{2}(T) T_{\min }^{\omega_{2}}$ is $580 \mathrm{~K}$ for $x=0$, which coincides with the temperature of the maximum of $\Gamma_{2}(T) . T_{\min }^{\omega_{2}}$ matches well the temperature of tetragonal-to-rhombohedral phase transition [4]. For $x=0.06$ and $0.08, T_{\min }^{\omega_{2}}$ is $\sim 420$ and $\sim 450 \mathrm{~K}$, respectively, while the excess in $\Gamma_{2}(T)$ is spread out over a large temperature range without a well-pronounced maximum. Here, $T_{\min }^{\omega_{2}}$ for $x=0.06$ and 0.08 is close to the corresponding depolarization temperature $T_{d}$ associated with the antiferroelectric-ferroelectric (AFE-FE) phase transition $[1,2,4]$.

Figure 3 shows the temperature dependencies of $\omega, \Gamma$, and the normalized integrated intensity $I$ of the phonon mode near $290 \mathrm{~cm}^{-1}$ dominated by vibrations of off-centered Ti. This mode splits into two components for all three compounds, and 


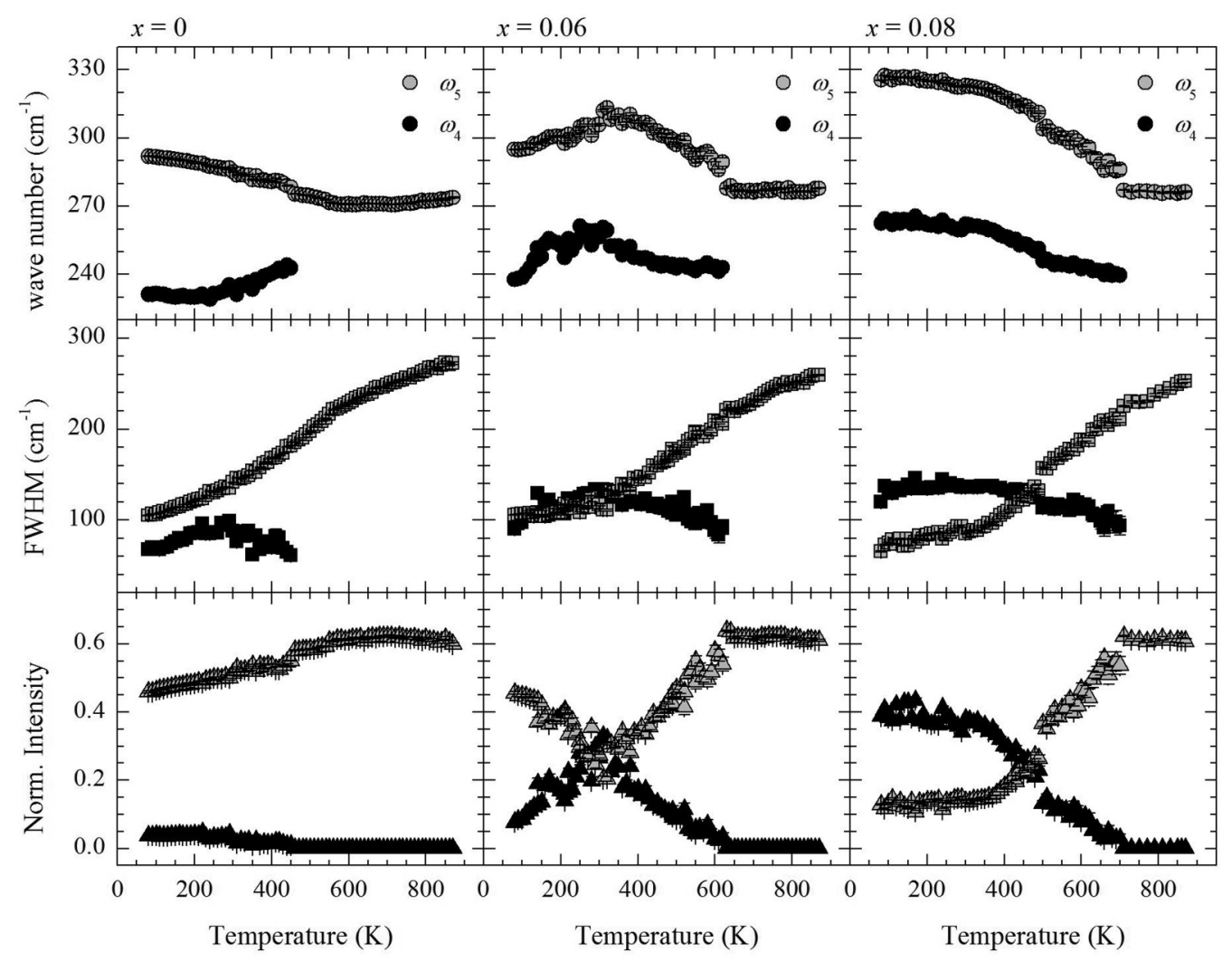

FIG. 3. Temperature evolution of the wave number $\omega$, FWHM, and intensity of Ti-localized mode in NBT- $x$ BT for $x=0,0.06$, and 0.08 ; gray and black symbols represent the higher energy and lower energy component, respectively.

the splitting temperature $T_{\mathrm{split}}^{\omega_{4}-\omega_{5}}$ increases with the increase in the Ba content from $460 \mathrm{~K}$ for $x=0$, to $630 \mathrm{~K}$ for $x=0.06$, and $705 \mathrm{~K}$ for $x=0.08$, which means that the addition of $\mathrm{Ba}$ stabilizes two distinct dynamic states of coupled off-centered Ti. However, the temperature evolution of the FWHMs and intensities of the two components of the Ti-localized mode reveals the turning point in this vibration state at $x=0.06 \sim$ $x_{\mathrm{MPB}}$. For $x=0<x_{\mathrm{MPB}}$, the higher energy component of the Ti mode (peak 5 in Fig. 1) has larger width and intensity than the lower energy component (peak 4 in Fig. 1) through the entire temperature range. For $x=0.08>x_{\mathrm{MPB}}, I_{4}(T)$ and $I_{5}(T)$ as well as $\Gamma_{4}(T)$ and $\Gamma_{5}(T)$ cross each other at $\sim 470 \mathrm{~K}$, and at low temperatures, the lower energy state becomes dominant. For $x=0.06 \sim x_{\mathrm{MPB}}$, the two components of the Ti-localized mode are very close to each other in both width and intensity over a temperature range 380-240 K, but at low temperatures, the higher energy state remains dominant; note that the upper temperature limit of $380 \mathrm{~K}$ is approximately the same as the splitting temperature of the Bi-localized mode $T_{\text {split }}^{\omega_{1}-\omega_{2}}$ for $x=0.06$.

Now let us consider the temperature dependencies of $\omega, \Gamma$, and $I$ of the $\mathrm{Bi}^{-\mathrm{TiO}_{3}}$ translation mode near $140 \mathrm{~cm}^{-1}$ (Fig. 4), which is composed of vibrations of both off-centered $\mathrm{Ti}$ and off-centered Bi. As can be seen in Fig. 4, there is a striking difference among the three compounds in the temperature behavior of this mode. For $x=0.06 \sim x_{\mathrm{MPB}}$, the $\mathrm{Bi}^{-\mathrm{TiO}_{3}}$ translation mode shows a tremendous softening and damping between 290 and $650 \mathrm{~K}$ with an extremum at $\sim 415 \mathrm{~K}$, which is close to the triple $\mathrm{FE}_{R 3 c}-\mathrm{AFE}-\mathrm{FE}_{P 4 m m}$ point in the $x-T$ diagram of NBT- $x$ BT $[1,2,4]$. This is a classical soft-mode behavior at the ferroelectric phase transition, responsible for the enhancement of the macroscopic static dielectric permittivity [37], and it clearly indicates that the abundant coupling between the $\mathrm{Bi}$ - and Ti-subsystems drives the material into a ferroelectric state. In strong contract, only a subtle hump near 420-450 K can be seen in the wave number, FWHM, and intensity of the $\mathrm{Bi}-\mathrm{TiO}_{3}$ mode for $x=0$, which should correspond to the AFE-FE transition, and a kink in $\omega_{3}(T)$ at $580 \mathrm{~K}$, which is close to the $P 4 b m-R 3 c$ phase-transition temperature $[1,2,4]$. For $x=0.08, \Gamma_{3}(T)$ and $I_{3}(T)$ exhibit a maximum near $450 \mathrm{~K}$, but it is not so well pronounced as for $x=0.06$, and more importantly, $\omega_{3}(T)$ continues to soften down to $80 \mathrm{~K}$, indicating instability in the system to the lowest temperature reached in our experiment. The gradual increase in the baseline of $\Gamma_{3}(T)$ on cooling, instead of the trivial decrease, is also indicative of significant instability induced by the temperature decrease for $x=0.08$.

Likewise to the Raman data, our x-ray PDF data indicate a high degree of structural resemblance in NBT- $x$ BT for $x$ below, near, and slightly above MPB in terms of first-neighbor distances. Figure 5 shows the low- $r$ range of PDFs of NBT$x \mathrm{BT}$ for $x=0,0.06$, and 0.08 in the range $1-5 \AA$, calculated from x-ray TES measured at different temperatures. In this $r$ range, the peaks can be assigned to specific A/B-O, A-B, and $\mathrm{A} / \mathrm{B}-\mathrm{A} / \mathrm{B}$ atom-atom correlations. However, it should be emphasized that the $\mathrm{x}$-ray data are greatly weighted by the heavy cations such as $\mathrm{Bi}^{3+}$, and hence, it is difficult to extract reliable information about $\mathrm{A} / \mathrm{B}-\mathrm{O}$ distances. In 


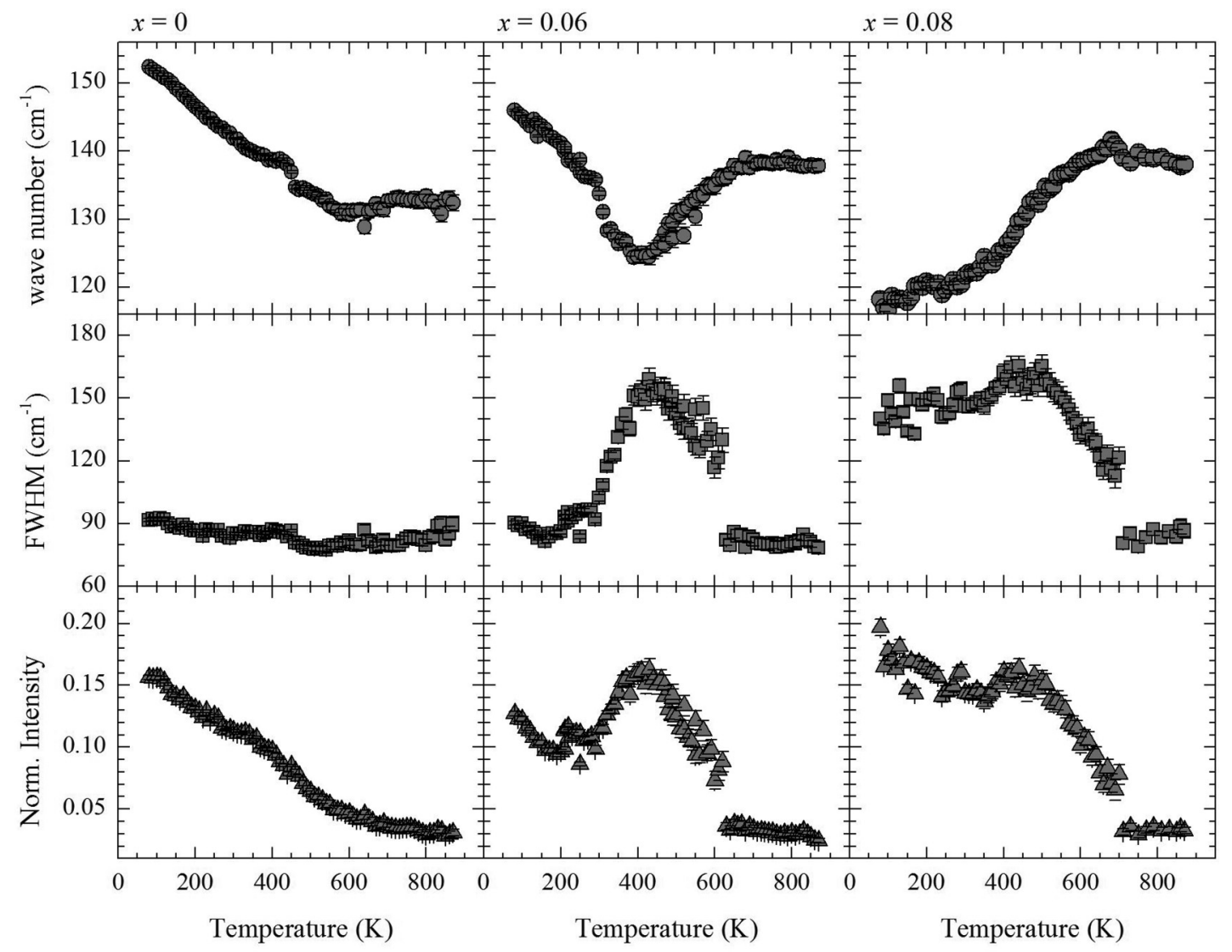

FIG. 4. Temperature evolution of the wave number, $\mathrm{FWHM}$, and intensity of $\mathrm{Bi}^{-\mathrm{TiO}_{3}}$ mode comprising vibrations of both $\mathrm{Bi}$ and $\mathrm{Ti}$ atoms.

addition, these PDFs, which have been obtained from fast in situ powder diffraction data, are not suitable for any rigorous model-based refinements [38]. Nevertheless, some qualitative observations on the cation-cation correlations can be performed. For example, the peak at $3.3 \AA$, which represents mainly A-B coherent distances, i.e. Ti-Bi/Na/ $\mathrm{Ba}$ distances, remains nearly unchanged both as a function of composition and temperature. The incorporation of $\mathrm{Ba}$ affects the PDF around $3.8 \AA$ measured at temperatures below the Curie temperature when $x$ is above MPB. This PDF feature is in general related to $\mathrm{A}-\mathrm{A}$ and $\mathrm{B}-\mathrm{B}$ correlations. Since $\mathrm{Bi}$ and $\mathrm{Ba}$ have much higher atomic numbers $Z$ than either Ti or
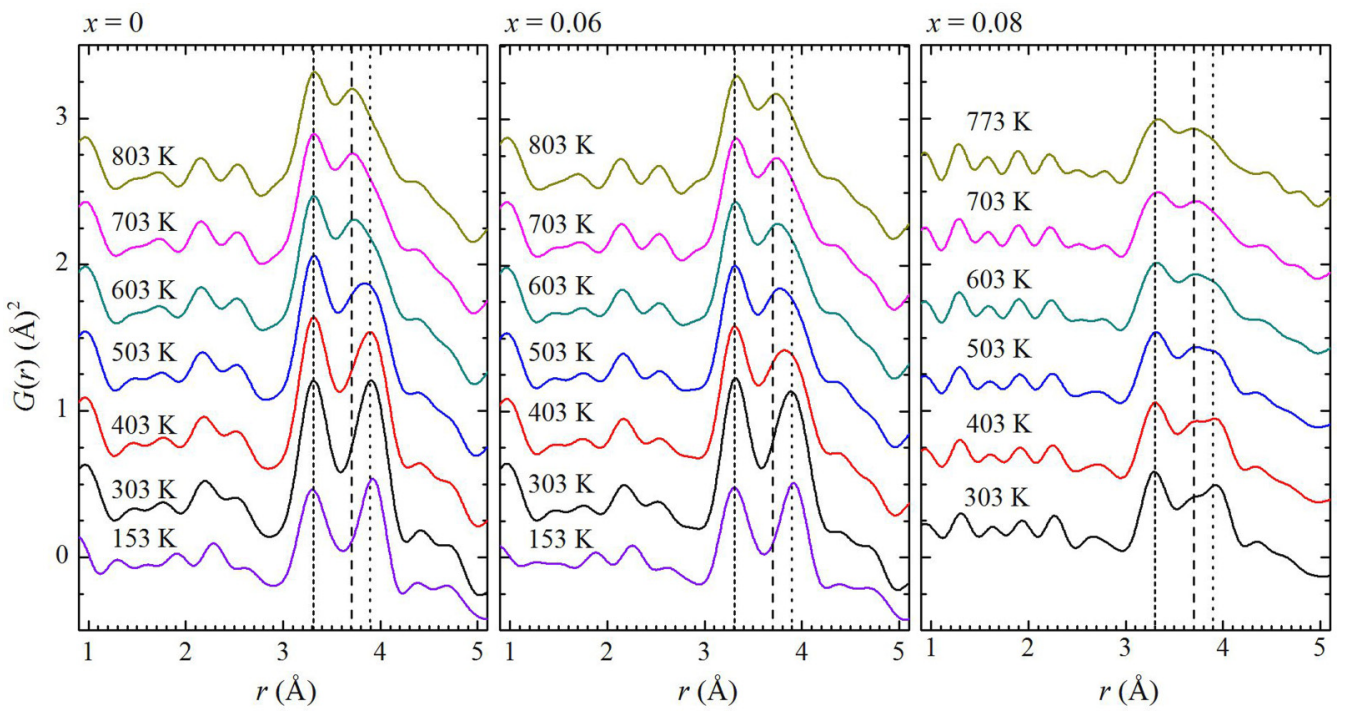

FIG. 5. (Color online) Temperature-dependent x-ray PDF patterns of NBT- $x$ BT for $x=0,0.06$, and 0.08 . The short-dashed lines (3.3 $\AA$ ) mark the feature corresponding to A-B distances; the dashed $(3.7 \AA)$ and dotted lines $(3.9 \AA)$ mark the two components in the feature related to $\mathrm{A}-\mathrm{A}$ and $\mathrm{B}-\mathrm{B}$ distances. 

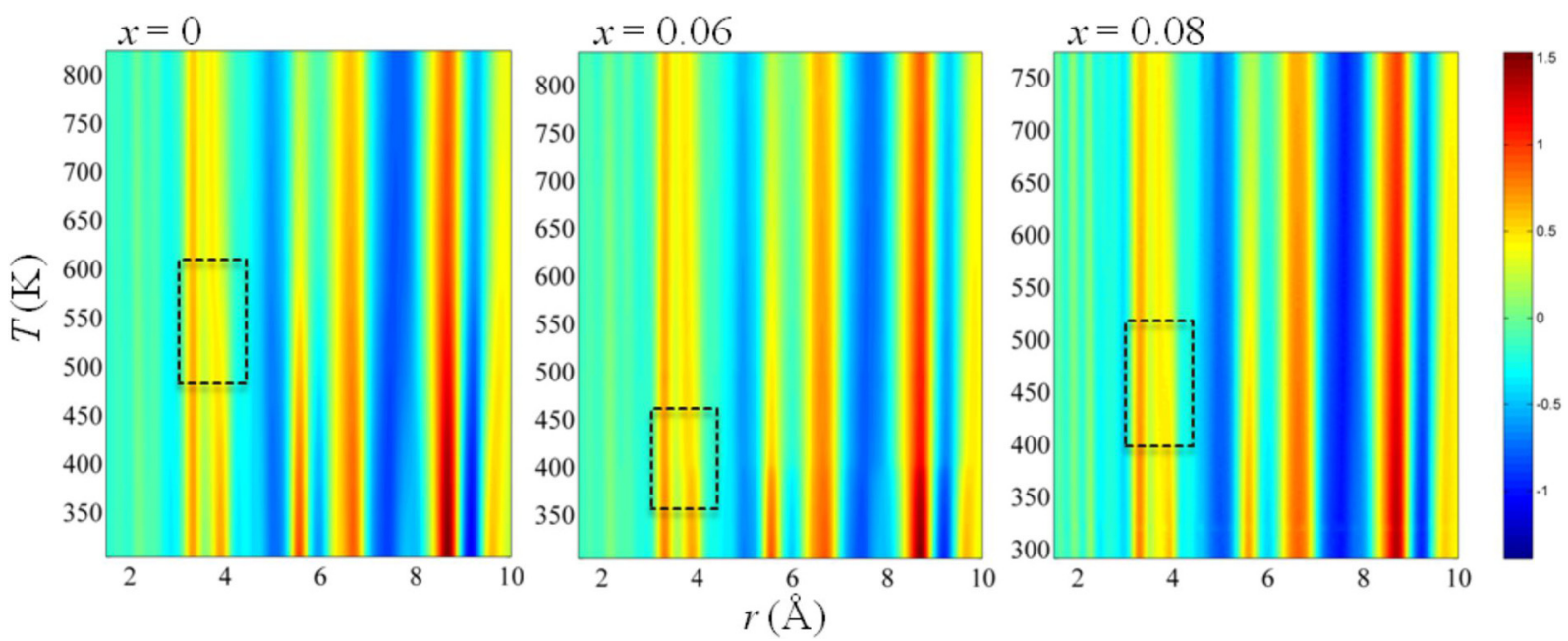

FIG. 6. (Color online) Temperature evolution of $\mathrm{x}$-ray PDF intensities for NBT- $x$ BT for $x=0,0.06$, and 0.08 ; the dashed rectangles mark the temperature ranges of the most pronounced changes in PDFs.

$\mathrm{Na}$, this PDF peak should primarily reflect $\mathrm{Bi}-\mathrm{Bi}, \mathrm{Bi}-\mathrm{Ba}$, and $\mathrm{Ba}-\mathrm{Ba}$ correlations. Furthermore, given the fact that the ratio between $\mathrm{Ba}$ content and $\mathrm{Bi}$ content is approximately 0.13 for NBT-0.06BT and 0.17 for NBT-0.08BT as well as that the $Z$ number of $\mathrm{Ba}$ is smaller than that of $\mathrm{Bi}$, it can be concluded that the dominant contribution to the PDF peak near $3.8 \AA$ comes primarily from $\mathrm{Bi}-\mathrm{Bi}$ coherent distances. Above $700 \mathrm{~K}$, this particular PDF range shows a peak at $3.7 \AA$ and a shoulder near $3.9 \AA$ for all three compounds with $x$ below, near, and above MPB. The shoulder near $3.9 \AA$ progressively increases in intensity with the temperature decrease for all three compounds, while the intensity of the peak near 3.7 $\AA$ decreases. The change in the intensity ratio is, however, considerably less for $x>x_{\mathrm{MPB}}$ than for $x<x_{\mathrm{MPB}}$ and $x \sim$ $x_{\mathrm{MPB}}$. This firmly reveals that, for $x>x_{\mathrm{MPB}}$, the incorporation of $\mathrm{Ba}$ prevents the vanishing of the shorter distanced $\mathrm{Bi}$ Bi correlations $(\sim 3.7 \AA)$ at temperatures below the Curie temperature, at least down to room temperature. For $x=0$ and 0.06 , the peak at $3.9 \AA$ continues to increase in relative intensity at temperatures below room temperature, suggesting diminishing dispersion in the off-centered Bi displacements on cooling. This result corroborates with the recent neutron PDF analysis of pure NBT [14] showing a strong dispersion of Bi displacements at $473 \mathrm{~K}$, which gradually disappears on cooling to $10 \mathrm{~K}$. Due to the limited beamtime for TES experiments, data below room temperature were not collected for $x=0$.

The temperature evolution of the PDF intensities in the range $1-10 \AA$ for $x=0,0.06$, and 0.08 can also be compared from the plots in Fig. 6, which provides a bird's eye view of the PDF patterns. Again, qualitatively, a temperature range can be identified where notable changes occur in the Bi-Bi correlations, suggesting a temperature-induced displacive-type phase transition for each composition. The temperature-driven alteration of PDF near $3.8 \AA$ is more gradual for $x=0.08$ compared to $x=0$ and 0.06 , which in fact follows the changes in the Raman scattering near $50 \mathrm{~cm}^{-1}$, revealing a splitting of the Bi-localized mode for $x=0$ and 0.06 , but not for $x=0.08$. Finally, the variation of the temperature range of the most pronounced PDF changes with $x$ resembles the trend in the reported $x-T$ phase diagram constructed by dielectric and polarization measurements $[1,2,4]$. This particular temperature range has been described as the crossover from antiferroelectric to ferroelectric order [1,2,39], and for pure NBT, it has been structurally characterized as the region of coexistence of several phases [9-11,32,33]. It is further important to note that, above this specific temperature range, the mesoscopic-scale (1-10 $\mathrm{A})$ correlations are consistently maintained up to $800 \mathrm{~K}$, which is in full accordance with the nonzero first-order Raman scattering of NBT- $x$ BT in the paraelectric state.

\section{CONCLUSIONS}

The temperature evolution of the Raman scattering near 50,140 , and $290 \mathrm{~cm}^{-1}$ suggests the following scenario of chemically induced structural phenomena in NBT- $x$ BT. The incorporation of $\mathrm{Ba}$ into the $\mathrm{A}$ site stabilizes the dynamical hopping of $\mathrm{B}$-site $\mathrm{Ti}$ and thus enhances the ferroelectric coupling between off-centered B-site cations. At the same time, Ba doping disturbs the coupling between off-centered $\mathrm{Bi}$, but for a certain value of Ba content, the delicate balance between coupled and uncoupled A-site cations allows for strong coupling between the $\mathrm{Bi}$ and $\mathrm{Ti}$ subsystems, i.e. dynamic coherence between the off-centered A- and B-site cations on the mesoscopic scale. This critical value $x_{c}$ corresponds to the so-called morphotropic phase. These mesoscopic-scale structural and dynamic phenomena may turn out to be one of the major reasons for the enhanced response functions near the MPB in NBT- $x$ BT.

\section{ACKNOWLEDGMENTS}

Parts of this research were carried out at the light source DORIS at DESY, a member of the Helmholtz Association (HGF). We would like to thank Martin v. Zimmermann for assistance in using beamline BW5. K.D. and R.B.N. are grateful to BMBF (Project 05K10WEB) for financial support. 
[1] T. Takenaka, K.-I. Maruyama, and K. Sakata, Jpn. J. Appl. Phys. 30, 2236 (1991).

[2] T. Takenaka, IEEE T. Ultrason. FERR 56, 1595 (2009).

[3] Y. Guo, Y. Liu, R. L. Withers, F. Brink, and H. Chen, Chem. Mater. 23, 219 (2011).

[4] W. Ge, H. Cao, C. DeVreugd, J. Li, and D. Viehland, J. Am. Ceram. Soc. 94, 3084 (2011).

[5] D. Maurya, M. Murayama, A. Pramanick, W. T. Reynolds, Jr., K. An, and S. Priya, J. Appl. Phys. 113, 114101 (2013).

[6] C. Ma, X. Tan, E. Dul'kin, and M. Roth, J. Appl. Phys. 108, 104105 (2010).

[7] J. Yao, N. Monsegue, M. Murayama, W. Leng, W. T. Reynolds, Q. Zhang, H. Luo, J. Li, W. Ge, and D. Viehland, Appl. Phys. Lett. 100, 012901 (2012).

[8] C. Ma, H. Guo, and X. Tan, Adv. Func. Mater. 23, 5261 (2013).

[9] W. Ge, C. P. Devreugd, D. Phelan, Q. Zhang, M. Ahart, J. Li, H. Luo, L. A. Boatner, D. Viehland, and P. M. Gehring, Phys. Rev. B 88, 174115 (2013).

[10] V. A. Isupov, Ferroelectrics 315, 123 (2005).

[11] M. Matsuura, H. Iida, K. Hirota, K. Ohwada, Y. Noguchi, and M. Miyayama, Phys. Rev. B 87, 064109 (2013).

[12] V. A. Shuvaeva, D. Zekria, A. M. Glazer, Q. Jiang, S. M. Weber, P. Bhattacharya, and P. A. Thomas, Phys. Rev. B 71, 174114 (2005).

[13] E. Aksel, J. S. Forrester, J. C. Nino, K. Page, D. P. Shoemaker, and J. L. Jones, Phys. Rev. B 87, 104113 (2013).

[14] D. S. Keeble, E. R. Barney, D. A. Keen, M. G. Tucker, J. Kreisel, and P. A. Thomas, Adv. Funct. Mater. 23, 185 (2013).

[15] A.-M. Welsch, B. J. Maier, B Mihailova, R. J. Angel, J. Zhao, C. Paulmann, J. M. Engel, M. Gospodinov, V. Marinova, and U. Bismayer, Z. Kristallogr. 226, 126 (2011).

[16] N. Waeselmann, B. Mihailova, B. J. Maier, C. Paulmann, M. Gospodinov, V. Marinova, and U. Bismayer, Phys. Rev. B 83, 214104 (2011).

[17] B. Mihailova, R. J. Angel, B. J. Maier, A.-M. Welsch, J. Zhao, M. Gospodinov, and U. Bismayer, IEEE T. Ultrason. FERR 58, 1905 (2011).

[18] B. Mihailova, G. Bogachev, V. Marinova, and L. Konstantinov, J. Phys. Chem. Solids 60, 1829 (1999).

[19] B. Mihailova, D. Toncheva, M. Gospodinov, and L. Konstantinov, Solid Stat. Commun. 112, 11 (1999).
[20] J. Petzelt, S. Kamba, J. Fabry, D. Noujni, V. Porokhonsky, A. Pashkin, I. Franke, K. Roleder, J. Suchanicz, R. Klein, and G. E. Kugel, J. Phys.: Condens. Matter 16, 2719 (2004).

[21] B. Wylie-van Eerd, D. Damjanovic, N. Klein, N. Setter, and J. Trodahl, Phys. Rev. B 82, 104112 (2010).

[22] D. Rout, K. S. Moon, V. S. Rao, and S. J. L. King, J. Appl. Phys. 108, 084102 (2010).

[23] L. Luo, W. Ge, J. Li, D. Viehland, C. Farley, R. Bodnar, Q. Zhang, and H. Luo, J. Appl. Phys. 109, 113507 (2011).

[24] J. Suchanucz, I. Jankowska-Sumara, and T. V. Krizina, J. Electroceram. 27, 45 (2011).

[25] G. Viola, H. Ning, X. Wei, M. Deluca, A. Adomkevicius, J. Khaliq, M. J. Reece, and H. Yan, J. Appl. Phys. 114, 014107 (2013).

[26] S. Zhang, X. Wang, and L. Li, Ferroelectrics 450, 28 (2013).

[27] D. Schütz, M. Deluca, W. Kraus, A. Feteira, T. Jackson, and K. Rechmann, Adv. Func. Mater. 22, 2285 (2012).

[28] A. P. Hammersley, S. O. Svensson, M. Hanfland, A. N. Fitch, and D. Hausermann, High Press. Res. 14, 235 (1996).

[29] P. Juhas, T. Davis, C. L. Farrow, and S. J. L. Billinge, J. Appl. Cryst. 46, 560 (2013).

[30] E. Kroumova, M. I. Aroyo, J. M. Perez-Mato, A. Kirov, C. Capillas, S. Ivantchev, and H. Wondratschek, Phase Transitions 76, 155 (2003).

[31] M. Gröting, S. Hayn, and K. Albe, J. Solid Stat. Chem. 184, 2041 (2011).

[32] G. O. Jones and P. A. Thomas, Acta Cryst. B 56, 426 (2000).

[33] E. Aksel, J. S. Forrester, J. L. Jones, P. A. Thomas, K. Page, and M. R. Suchomel, Appl. Phys. Lett. 98, 152901 (2011).

[34] K. Datta, P. A. Thomas, and K. Roleder, Phys. Rev. B 82, 224105 (2010).

[35] R. Loudon, Adv. Phys. 13, 423 (1964).

[36] J. Kreisel, A. M. Glazer, G. Jones, P. A. Thomas, L. Abello, and G. Lucazeau, J. Phys.: Condens. Matter 12, 3267 (2000).

[37] R. Blinc and B. Žekš, Soft Modes in Ferroelectrics and Antferroelectrics (Elsevier, North-Holland, 1974).

[38] T. Egami and S. J. L. Billinge, Underneath the Bragg Peaks: Structural Analysis of Complex Materials (Elsevier, Kidlington, 2003).

[39] Y. Hiruma, H. Nagata, and T. Takenaka, J. Appl. Phys. 105, 084112 (2009). 